\title{
Optimized Lattice-based 16-level Subcarrier Modulation for IM/DD Systems
}

\author{
Krzysztof Szczerba $^{(1)}$, Johnny Karout ${ }^{(2)}$, Magnus Karlsson ${ }^{(1)}$, Peter A. Andrekson ${ }^{(1)}$, Erik Agrell ${ }^{(2)}$ \\ (1) Department of Microtechnology and Nanoscience, Photonics Laboratory, Chalmers University of \\ Technology, 412-96 Gothenburg, Sweden, krzysztof.szczerba@chalmers.se \\ (2) Department of Signals and Systems, Chalmers University of Technology, 412-96 Gothenburg, \\ Sweden.
}

Abstract We present an experimental demonstration of an optimized 16-level lattice-based singlecycle subcarrier modulation for IM/DD systems at 10 Gbps. The new format has $2.5 \mathrm{~dB}$ better sensitivity than single-cycle subcarrier 16-QAM and up to $1 \mathrm{~dB}$ better sensitivity than baseband 4-PAM at the same bit rate.

\section{Introduction}

The increasing popularity of cloud computing and demand for larger datacenters is currently a driving factor for a development of high-speed short-range optical interconnects. Such optical links are usually built with low-cost and highspeed vertical cavity surface emitting lasers (VCSELs). Intensity modulation and direct detection (IM/DD) is used in such systems because of cost constraints. The prevalent modulation format in commercial links is on-off keying (OOK).

Recently, research on more spectrally efficient modulation formats has become very active. Modulation formats such as a singlecycle subcarrier 16-level quadrature amplitude modulation (QAM) and a baseband 4-level pulse amplitude modulation (PAM) have been demonstrated at, respectively, $37 \mathrm{Gbps}^{1}$ and $30 \mathrm{Gbps}^{2}$. A big advantage of PAM is its simplicity, allowing real-time and high-speed implantations. Real-time, high-speed implementations of subcarrier QAM are difficult because of its complexity. On the other hand, carrier-less amplitude and phase modulation (CAP), which also uses in-phase and quadrature pulses as basis functions, has been demonstrated experimentally at $40 \mathrm{Gbps}$ in real time $^{3}$. Transversal filters were used to generate the CAP signal ${ }^{3}$. Orthogonal multi-pulse modulation with three orthogonal basis functions has also been demonstrated in real-time using the same method $^{4}$.

Most multi-level modulation formats have a disadvantage of reduced sensitivity, compared to OOK. Theoretically, $4.8 \mathrm{~dB}$ more optical power is required to double the bit rate by using baseband 4-PAM instead of OOK at the same symbol rate $^{2}$. The sensitivity of modulation schemes is an important consideration because the optical power budget is limited by factors such as limited output power of VCSELs, power

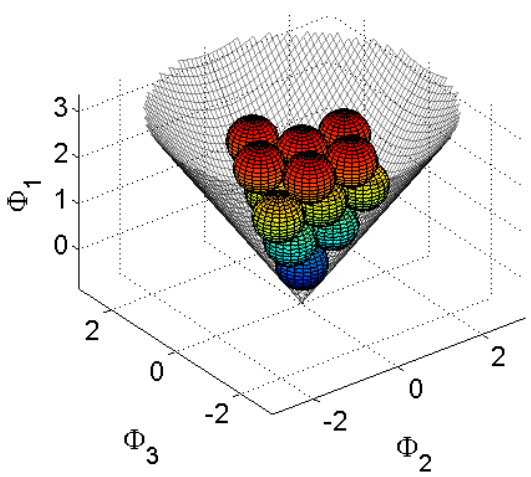

Fig. 1: Constellation diagram of a 16-level singlecycle subcarrier format based on face centered cubic lattice $\left(\mathrm{L}_{16}\right)$.

handling abilities of detectors, losses, or eye safety constraints.

Three dimensional single-cycle subcarrier modulation formats

The two dimensions (in-phase and quadraturephase, denoted here $\Phi_{2}$ and $\Phi_{3}$ ) of the singlecycle subcarrier modulation can be complemented with a third orthogonal dimension, represented by a rectangular pulse

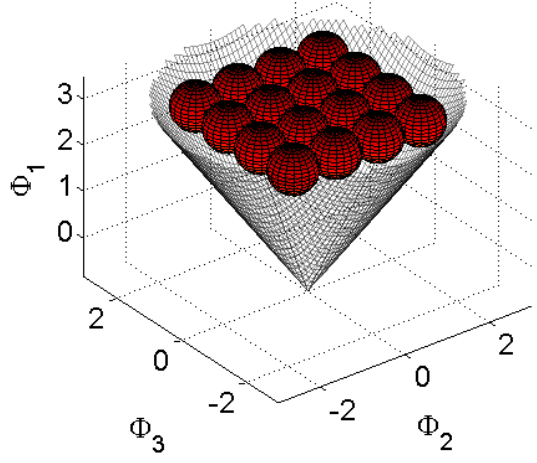

Fig. 2: Subcarrier 16-QAM constellation represented in a three dimensional signal space. 


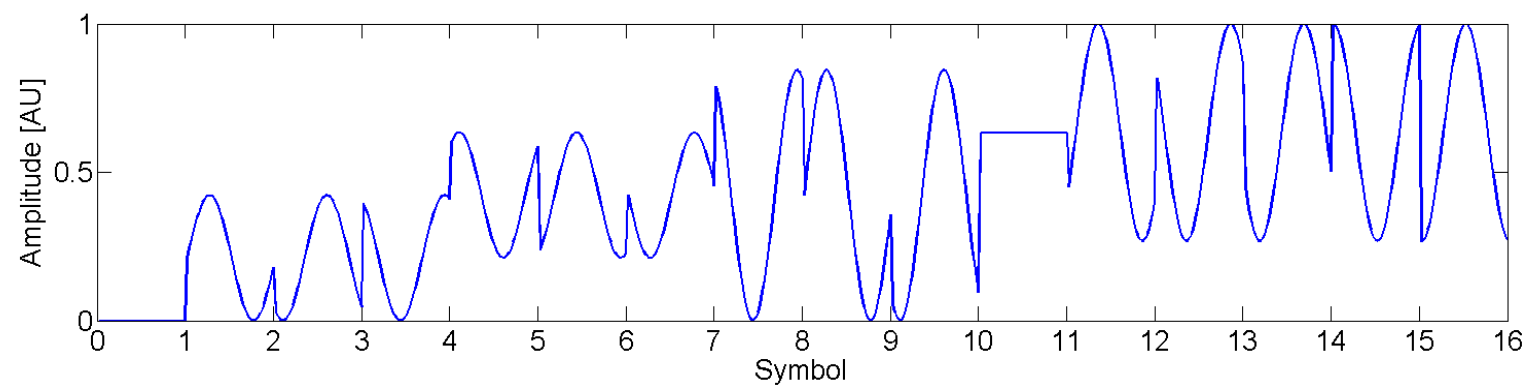

Fig. 3: Symbols of the $L_{16}$ modulation presented in time domain.

of the same duration as the symbol period (denoted $\Phi_{1}$ ). It can be thought of as an adaptive bias for the subcarrier symbols. The resultant three-dimensional signal space was analyzed in ${ }^{7}$.

For IM/DD systems with directly modulated lasers, any signal modulating the laser must be non-negative to avoid clipping, and the electrical subcarrier has be biased to make it nonnegative. In the simplest case, the subcarrier signal is shifted by the same amount for all symbols, even when for some symbols a lower bias would suffice. Consequently, some of the optical power does not carry information, because the modulation depth is lower.

The $\Phi_{1}$ dimension allows for so called "adaptive biasing", which can improve the sensitivity of the subcarrier modulation ${ }^{5}$ and was demonstrated experimentally ${ }^{6}$ for 8 -level singlecycle subcarrier modulation. Modulation formats with sensitivity better than PAM at the same spectral efficiency were proposed $i^{8}$. For example, at the spectral efficiency of $2 \mathrm{bits} / \mathrm{s} / \mathrm{Hz}$ and at the same bit rate, sensitivity better by up to $1.5 \mathrm{~dB}$ than 4-PAM can be achieved with an optimized 16-level subcarrier modulation ${ }^{8}$. The constellation shape is irregular in this case. It is also possible to perform an optimization of lattice-based constellations, which results in more regular shapes. The face-centered cubic (FCC) lattice provides the best packing of symbols in a three dimensional space. A format based on the FCC lattice was proposed in $^{8}$ (denoted as $L_{16}$ ), with an asymptotical sensitivity $1 \mathrm{~dB}$ better than baseband 4-PAM and $2.5 \mathrm{~dB}$ better sensitivity than single-cycle subcarrier 16QAM. The theoretical performance gains are valid for systems with additive white Gaussian noise (AWGN). The symbols of $L_{16}$ were given in $^{8}$. Using $a=\sqrt{ }(2 / 3), b=1 / \sqrt{3}$, and $c=\sqrt{3} / 6$ the symbols in $L_{16}$ can be defined in the $\left\{\Phi_{1}, \Phi_{2}\right.$ $\Phi_{3\}}$ signal space as follows: $\{(0,0,0),(a, 0, b)$, $(a, \pm 1 / 2,-c), \quad(2 a, \pm 1 / 2, c), \quad(2 a, 0,-b)$, $(2 a, \pm 1,-b), \quad(2 a, 0,2 b), \quad(3 a, 0,0), \quad(3 a, \pm 1,0)$, $(3 a, \pm 1 / 2,-3 c),(3 a, 1 / 2,3 c)\}$.

An illustration of the $L_{16}$ constellation in three dimensions is presented in Fig. 1, with spheres to visualize the locations of symbol. The spheres have a diameter of one minimum distance between the symbols. The symbols are located at the centers of the spheres. The cone in Fig. 1 illustrates the admissible signal space defined by the non-negativity constraint, i.e. the part of the signal space which gives symbols with nonnegative waveforms ${ }^{7}$. For comparison the subcarrier 16-QAM constellation is shown in the same signal space in Fig.2. Obviously $L_{16}$ utilizes the admissible signal space in a more efficient way. An illustration of an $L_{16}$ symbol waveform in time domain is shown in Fig. 3.

\section{Experimental setup}

Three modulation formats with the same spectral efficiency were experimentally compared, $\mathrm{L}_{16}$, baseband 4-PAM and 16-QAM with a single-cycle subcarrier. All formats were compared at $10 \mathrm{Gbps}$, which means that 4-PAM was operated at 5 Gbaud and $L_{16}$ and subcarrier 16-QAM were operated at 2.5 Gbaud, since the bandwidth of the single-cycle subcarrier signal is twice the symbol rate ${ }^{1}$. The electrical bandwidth of all signals was $5 \mathrm{GHz}$, counted to the first spectral null. The modulation formats were tested in a link consisting of a directly modulated VCSEL operating at a wavelength of $850 \mathrm{~nm}$ with a $16 \mathrm{GHz}$ modulation bandwidth, multimode fiber patch-cord and a photoreceiver with a 12 $\mathrm{GHz}$ modulation bandwidth, for direct detection. The VCSEL was from the same batch as the one reported in $^{9}$. All modulation formats were generated using an arbitrary waveform generator and after transmission detected and sampled using a real time sampling oscilloscope and further processed off-line on a personal computer to obtain the symbol error rates (SER). Because of the off-line processing the lowest SER values obtainable with reasonable effort were around $10^{-5}$. The modulator and demodulator for $L_{16}$ were based on the classical I/Q structure for ordinary QAM and PSK formats, but with an extra branch for the third dimension, which represents only a small increase in complexity over usual subcarrier modulator or 


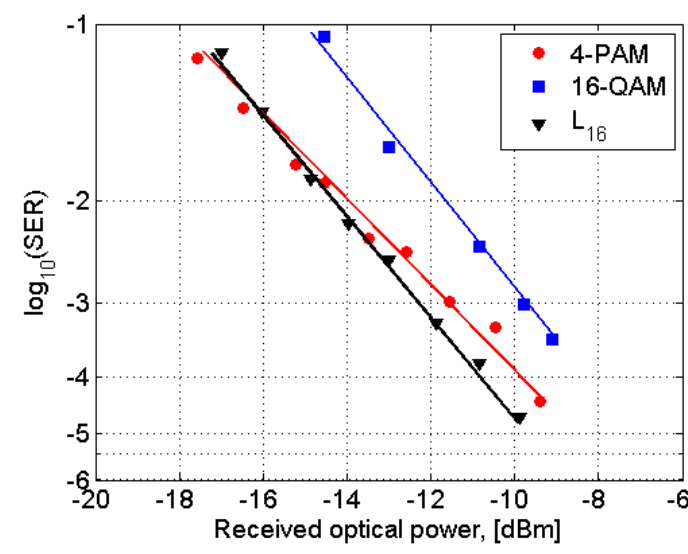

Fig. 4: Experimental bit-error rates for different $2 \mathrm{bit} / \mathrm{s} / \mathrm{Hz}$ formats in an IM/DD VCSEL link

demodulator. In the off-line receiver a maximum likelihood detector was used, since it minimizes the SER for AWGN channels.

\section{Results and discussion}

The experimentally obtained SER values for a back to back (BTB) case are presented in Fig. 4. The bit error rate would be dependent on the bit-labeling of the symbols. The optimal labeling for $L_{16}$ remains to be found. An example of a received three-dimensional constellation diagram of $L_{16}$ is illustrated in Fig. 5 .

In the BTB configuration, the sensitivity of the $L_{16}$ format is almost $1 \mathrm{~dB}$ better than 4-PAM at SER of $10^{-4}$ and at $2.5 \mathrm{~dB}$ than single-cycle subcarrier 16-QAM. This is agreement with the theoretical expectations from ${ }^{8}$.

There are few remarks on the observed results. At SER above $10^{-2}$ the performance of 4-PAM and $L_{16}$ is similar. In theory the sensitivity gain of $L_{16}$ over 4-PAM is increasing to the asymptotical value of $1 \mathrm{~dB}$ for low SER ${ }^{8}$. The experimentally demonstrated sensitivity gain of

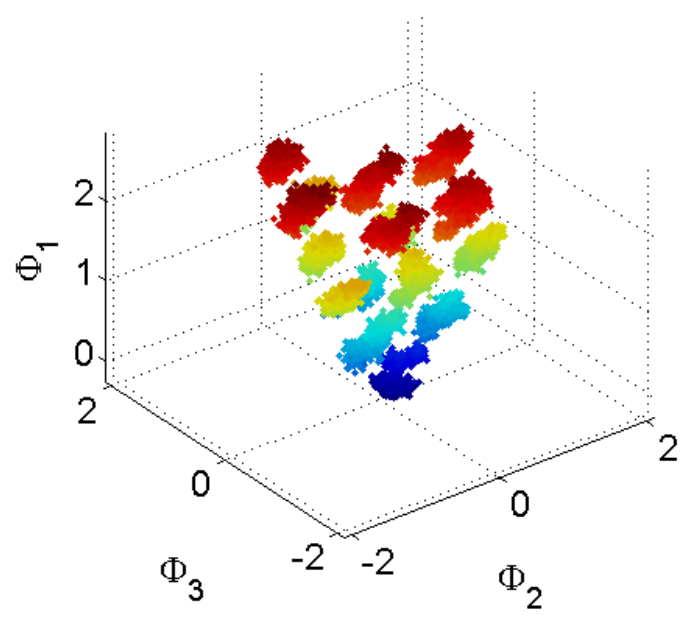

Fig. 5: Constellation diagram of $L_{16}$ at $-11 \mathrm{dBm}$ received optical power. The different $\Phi_{1}$ levels are shown with different colors.
$\mathrm{L}_{16}$ over 4-PAM is increasing to almost $1 \mathrm{~dB}$. Finally, although the transmission bit rate of the presented modulation formats is $10 \mathrm{Gbps}$, for practical data transmission forward error correction (FEC) would have to be used at the observed SER. With 7\% FEC overhead the usable bitrate is $9.3 \mathrm{Gbps}$.

\section{Conclusions}

We have experimentally demonstrated an optimized lattice-based 16-level singlesubcarrier modulation format with $1 \mathrm{~dB}$ better sensitivity than 4-PAM modulation, at $10 \mathrm{Gbps}$ data rates.

Although the presented work was done with off-line processing, it should be possible to implement it in real time, in a manner similar to the CAP modulation ${ }^{3}$. The somewhat higher complexity of the $\mathrm{L}_{16}$ compared to the singlecycle subcarrier 16-QAM, is traded for $2.5 \mathrm{~dB}$ sensitivity gain.

Potential applications of the $L_{16}$ format, beyond IM/DD systems with VCSELS and MMF could include for example polymer optical fiber networks or wireless optical transmission with IM/DD.

\section{Acknowledgements}

This work was supported by the Swedish Foundation for Strategic Research (SSF) and we would like to kindly thank for their support.

\section{References}

[1] K. Szczerba et al., Proc. ECOC'10, We.7.B.2 (2010).

[2] K. Szczerba et al., Opt. Express 19, B203B208 (2011).

[3] J. D. Ingham et al., Proc. OFC'11, OThZ3 (2011).

[4] J. D. Ingham et al., Proc. ECOC'11, Tu.3.C.2 (2011).

[5] S. Hranilovic et al., Proc. ISCAS'99, 338341, (1999).

[6] K. Szczerba et al., Proc. ECOC'11, We.10.P1.117 (2011).

[7] S. Hranilovic et al., IEEE Trans. Inf. Theory, 49, 1385-1399, (2003).

[8] J. Karout et al., to appear in IEEE Trans. Inf. Theory, available: http://arxiv.org/abs/1106.2819.

[9] P. Westbergh et al. Electron. Lett., 45, 366368, (2009). 